\title{
Review Article \\ Prevalence of Dietary Supplement Use in Patients with Proven or Suspected Cardiovascular Disease
}

\author{
Yu Sun Bin ${ }^{1}$ and Hosen Kiat ${ }^{1,2}$ \\ ${ }^{1}$ Cardiac Health Institute, 173 Shaftsbury Road, Eastwood, NSW 2122, Australia \\ ${ }^{2}$ School of Advanced Medicine, Macquarie University, NSW 2109, Australia
}

Correspondence should be addressed to Hosen Kiat, hosen.kiat@chi.org.au

Received 27 April 2010; Revised 14 June 2010; Accepted 10 July 2010

Copyright (C) 2011 Y.S. Bin and H. Kiat. This is an open access article distributed under the Creative Commons Attribution License, which permits unrestricted use, distribution, and reproduction in any medium, provided the original work is properly cited.

\begin{abstract}
Systematic search of bibliographic databases was conducted to describe the prevalence of dietary supplement use in cardiac patients. Included for review were studies that investigated supplement use in people with cardiovascular risk factors or proven cardiovascular disease. Databases searched were Medline, EMBASE, CINAHL, AMED, Meditext, H\&S and IPA. Over five hundred articles were retrieved and twenty studies met the criteria for this review. Dietary supplements were taken by a median $36 \%$ (interquartile range: $26-42 \%$ ) of cardiac patients; $36 \%$ (IQR $18-43 \%$ ) reported taking a vitamin/mineral supplement and $12 \%$ (IQR 7-21\%) used herbal supplements. Many users indicated that supplements were taken specifically for heart health and 16-64\% of users reported using supplements alongside prescription medications. However 39-95\% of treating physicians were unaware of patients' supplement use. Dietary supplement use in patients with cardiovascular disease appears common, as does the concurrent use of supplements with prescription medicines. This information is often not communicated to doctors and treating physicians may need to be more proactive in asking about supplement use.
\end{abstract}

\section{Introduction}

A dietary supplement is defined as "any product intended for ingestion as a supplement to the diet" [1]. These substances include amino acids, charcoal, choline salts, essential oils, plant or herbal material, homeopathic preparations, nonvaccine microorganisms, minerals, nonhuman animal material, lipids, substances from bees, and vitamins or provitamins. In this paper, we use the term "dietary supplement" to indicate any of these substances used as complementary and alternative medicine (CAM) and taken orally for the improvement of health or the prevention of illness.

The use of dietary supplements is common; in the general population, dietary supplements are the second most commonly used type of CAM after prayer [2]. Dietary supplements can interfere with the biotherapeutic action of prescription medications, and this is of particular concern in cardiac patients, many of whom are on long-term medications and are at increased risk of acute life-threatening events. Chronic conditions such as arthritis, cancer, depression, and anxiety have been consistently associated with CAM use [3], so it is probable that patients with chronic cardiovascular disease are also likely to use CAM. We conducted a systematic review of the literature to find the prevalence of dietary supplement use by cardiac patients and to identify commonly used supplements.

\section{Method}

2.1. Study Selection. A literature search was carried out on 2 December 2009. We searched the following databases from their earliest availability up to and including November 2009: Medline through OvidSP from 1950, EMBASE through embase.com from 1980, CINAHL through EBSCO Host from 1982, Allied and Complementary Medicine (AMED) through OvidSP from 1985, Australian Medical Index (Meditext) through Informit from 1968, Health \& Society (H\&S) through Informit from 1980, and International Pharmacy Abstracts (IPA) through OvidSP from 1970.

$\mathrm{MeSH}$ keywords and qualifiers were used for databases indexed using this method, that is, Medline and CINAHL. 


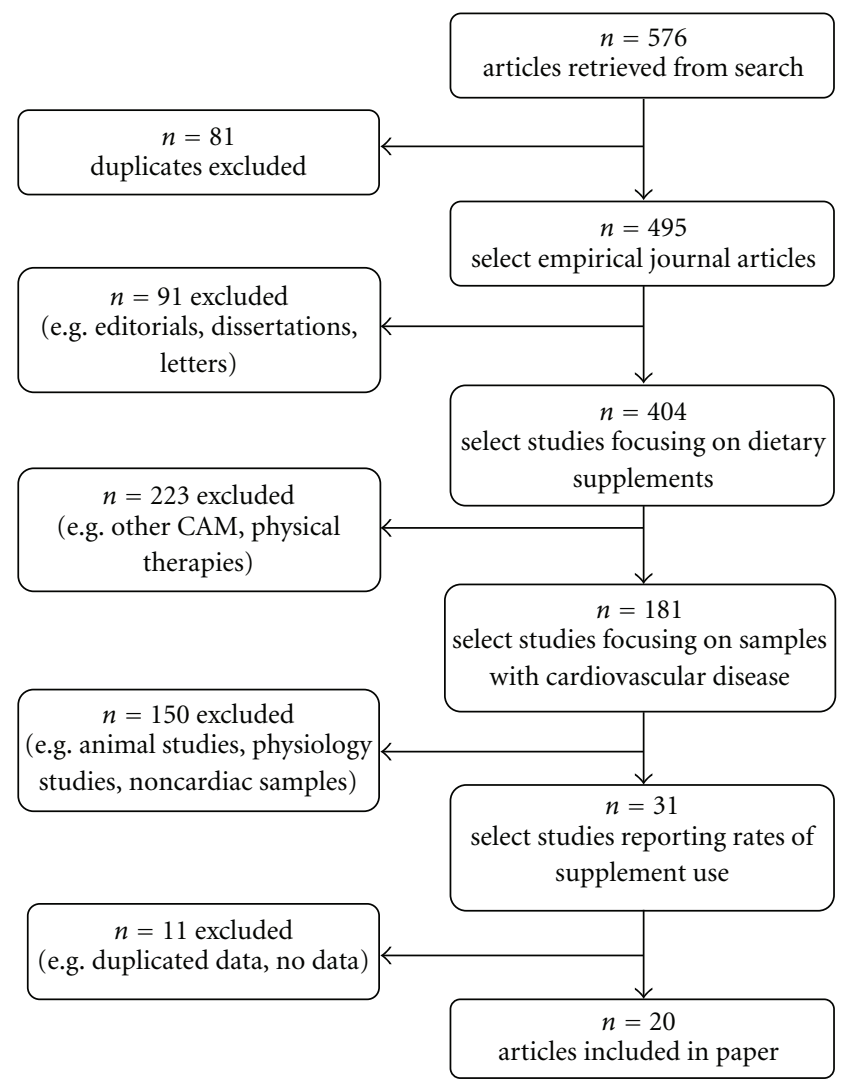

FIGURE 1: Flowchart showing the selection of studies from search results.

We used the MeSH terms "cardiovascular diseases" with the qualifier "/therapy" to find articles relevant to treatment of cardiovascular disease. To find articles on dietary supplement use, we used the MeSH terms "medicine, traditional", "dietary supplements", "vitamins", "minerals", each qualified with "/utilization". All MeSH terms were exploded to include subheadings. The "cardiovascular disease" search results were then intersected with each of the dietary supplement searches. For databases not indexed using $\mathrm{MeSH}$, searches were performed using the same terms without the qualifiers. In these databases, additional searches were conducted using the terms "cardiovascular diseases", "cardiology", and "cardiac" intersected with each of "complementary and alternative medicine", "dietary supplement", and "herbal medicine". Search results were limited to articles in English and those that dealt with humans where these options were available.

The titles and abstracts of articles were read and refined to include only those studies that (i) reported the prevalence of dietary supplement use, (ii) involved a sample of patients either attending for cardiac care or those reporting a cardiovascular condition, and (iii) indicated the types of supplements used. If abstracts were not available or were ambiguous with regard to these criteria, the full-text article was obtained for inspection. Articles were excluded if (i) study methods were not described, (ii) prevalence data on dietary supplement use were not reported (or these could not be calculated from reported data), and if (iii) they were not in English. Citations in the relevant papers were also used to locate articles not found by the above methods. Figure 1 shows the selection of studies from the search results.

2.2. Data Extraction. Two data tables were designed for systematic extraction of study information. Methodological details were extracted and compared in Table 1, while study content and results were extracted and summarised in Table 2. The most common supplements are listed in Table 3, and for the sake of brevity, this only includes supplements used by $\geq 1 \%$ of patients and which were reported in 2 or more of the included studies.

2.3. Statistical Analyses. Prevalence of overall supplement use was obtained from each study. For studies that did not report the overall prevalence, the figure corresponding to the most common supplement or most common supplement category was used. This method is expected to underestimate rather than overestimate the total number of supplement users. The median prevalence across studies and the corresponding interquartile ranges (IQR) were calculated for (i) any supplement use, (ii) the use of vitamin/mineral supplements, and (iii) the use of herbal supplements. Where studies reported use of individual supplements, data was extracted and pooled figures are presented in Table 3. 


\section{Results}

3.1. Study Characteristics. Of the 576 studies found, 22 studies met the inclusion criteria. Two of these were excluded $[4,5]$ because they analysed subsets of the same data as another study [6]. Therefore, 20 studies were included for review [6-25].

All included studies were conducted between 1995 and 2008. Eleven were from the United States $[6,8,9,11-$ $14,17,18,21,25]$, three were from Canada $[7,19,23]$ and the others were from Hong Kong [22], India [20], Italy [15], Nigeria [10], Turkey [24], and the United Kingdom [16]. Three studies analysed data from population surveys in the US $[6,11,13]$, one contacted a registry of patients with cardiovascular disease [23], and the others sampled consecutive patients by convenience from their respective hospital, outpatient, or specialist cardiology clinic [7-10, 12, 14-22, 24, 25]. Of the studies involving clinical samples, five collected data by way of a patient-completed survey [7, 17$19,25]$, seven conducted face-to-face interviews $[10,12,14-$ $16,20,24]$, two used a combination of telephone and faceto-face interviews $[8,9]$, and two drew on information from routine clinical examination $[21,22]$.

Thoroughness of methodological reporting varied between studies (Table 1). Only three of the 16 clinical studies commented on the representativeness of their sample compared to the greater clinical population $[7,19,23]$. Six did not report the survey response rate $[9,10,14,19,21,24]$. For those that did, responding ranged from 26\%-100\% (mean 74\%). Only four of the clinical studies piloted their survey instrument $[7,19,24]$ and two trained multiple interviewers to an equivalent standard [12, 23]. Four performed a sample-size calculation to determine whether there was sufficient statistical power to detect differences between supplement users and nonusers [8, 14, 23, 25]. Many studies did not explicitly state what was meant by "dietary supplement" or CAM and three studies did not define the time period over which supplement use was surveyed $[9,10,23]$.

Six out of 20 studies surveyed the use of CAM as well as dietary supplements $[6,8,10,12,20,23]$, which included physical therapies such as chiropractic and yoga, and mindbody therapies such as relaxation and hypnosis. Another four included the use of nonprescription medications as well as dietary supplements $[7,9,11,19]$. Only results specific to dietary supplements are reported in this paper.

3.2. Prevalence of Dietary Supplement Use. Any supplement use was reported by a median 36\% (IQR 26\%-42\%) of patients across all 20 studies. Vitamins and minerals were taken by a median 36\% (IQR 18\%-43\%) of patients across 17 studies. Herbal substances were taken by a median 12\% (IQR $7 \%-21 \%$ ) of patients across all 20 studies. Other supplements (fish oil, glucosamine, homeopathy, etc.) were taken by a median $7 \%$ of patients (IQR 5\%-10\%) across 14 studies.

We also analysed results with respect to time periods surveyed. Seven studies looked at ever or yearly use, finding a median $25 \%$ of respondents used supplements (IQR $17 \%-$ $45 \%$ ). Three or 6-month use was surveyed by four studies with median 49\% users (IQR 37\%-61\%). Median use within the last month in six studies was found to be 39\% (IQR 37\%$40 \%$ ). Only two studies investigated the duration of supplement use, which averaged 39 months [24], with $88 \%$ of herbal users taking the supplement for a month or more [13].

Supplement users tended to use more than one supplement at a time: two studies reported an average of 2 supplements per user $[14,24]$, while another recorded an average of 3 supplements per user [21]. Common supplements used by cardiac patients are summarized in Table 3 with the most common by far being multivitamins and minerals.

3.3. Concomitant Use of Supplements and Prescription Medications. Five studies reported data on the concomitant use of prescription medication and dietary supplements $[11,13$, $17,20,22,23$ ]. The study of Indian patients indicated $64 \%$ were using CAM in conjunction with prescription antihypertensives, although $86 \%$ had started conventional treatment first, while 9\% had began CAM first and 5\% had started both alternative and prescription treatments simultaneously [20]. One population survey in the US indicated that $36 \%$ of vitamin/mineral users and $16 \%$ of other supplement users used prescription medication for cardiovascular health [11], while another found that $63 \%$ of those with hypertension and $64 \%$ of those with high cholesterol were found to also take dietary supplements with their medications for cardiovascular disease [13]. Thirteen percent of Canadian patients with diagnosed coronary artery disease (CAD) had used a dietary supplement in conjunction with warfarin, amiodarone, sotalol, or digoxin [23], while $26 \%$ of Chinese patients on warfarin had taken a herbal medicine in the previous week [22]. Supplement users were as likely as nonusers to be taking aspirin, betablockers, ACE inhibitors, statins, and warfarin [17]. There was no difference between herb users and nonusers in terms of indication for warfarin, and the duration and dosage of warfarin therapy [22].

Three studies looked at the impact of supplement use on compliance $[10,15,16]$. Of the two which surveyed hypertensive patients $[10,16]$, one found no difference between supplement users and nonusers on adherence to antihypertensive medication [10], while the other found that female supplement users had lower adherence to prescription medications [16]. The third study that investigated outpatients with heart failure reported that 1 of 22 herbal supplement users reduced or interrupted heart failure medications while taking herbal medicine [15].

3.4. Cardiovascular Health and Supplement Use. In patients with heart failure, up to $82 \%$ of supplement users were taking a supplement specifically for cardiovascular health [25]. In patients with diagnosed cardiovascular disease, 18 out of 42 supplements (43\%) were taken for a cardiac reason [14].

Supplement users were more likely than nonusers to be under specialist cardiology care [9], although the use of herbal supplements was not associated with previous myocardial infarction or previous percutaneous coronary intervention in a Turkish sample [24]. A US study [21] 


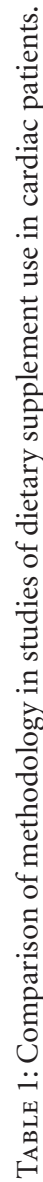

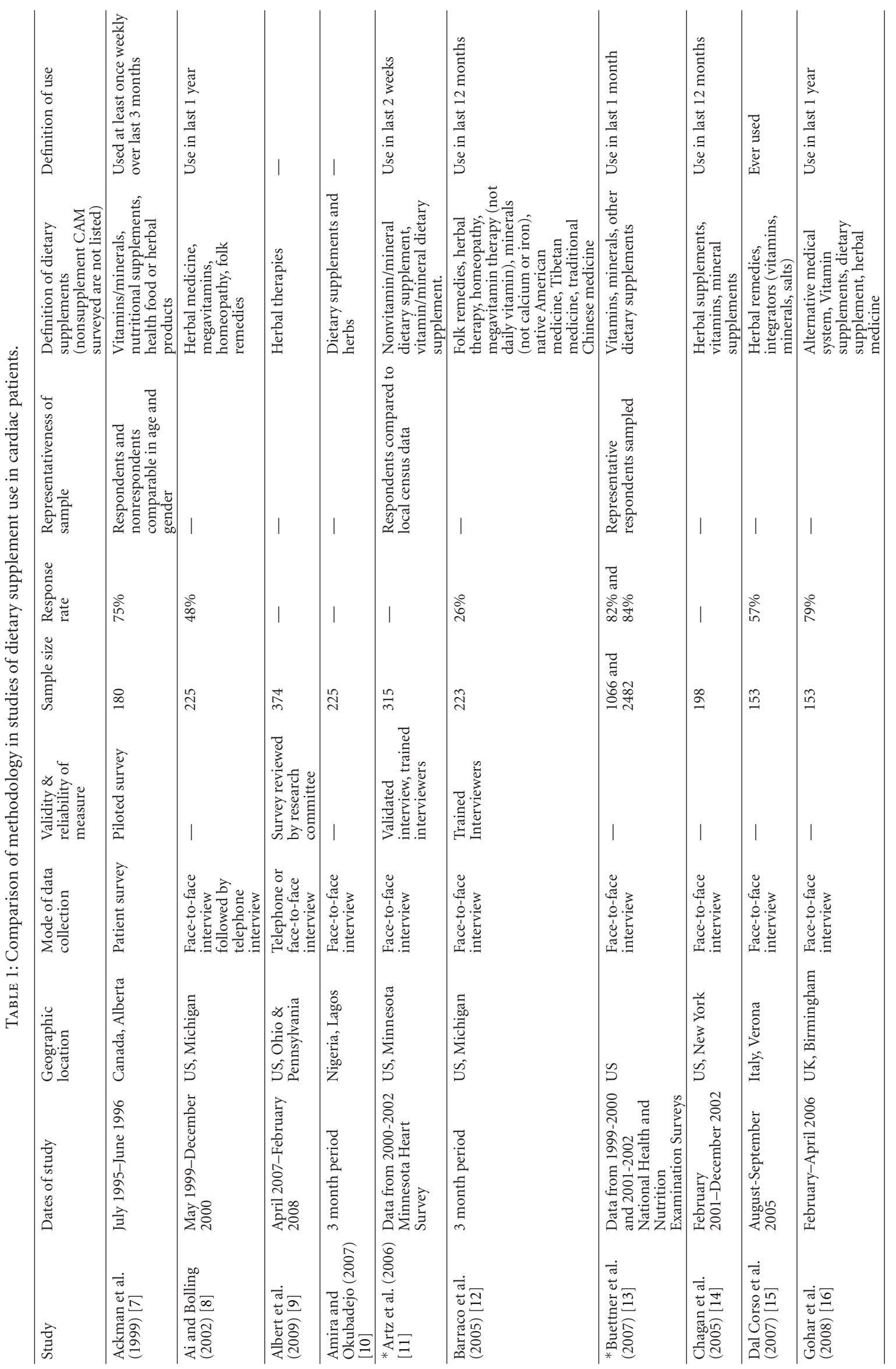




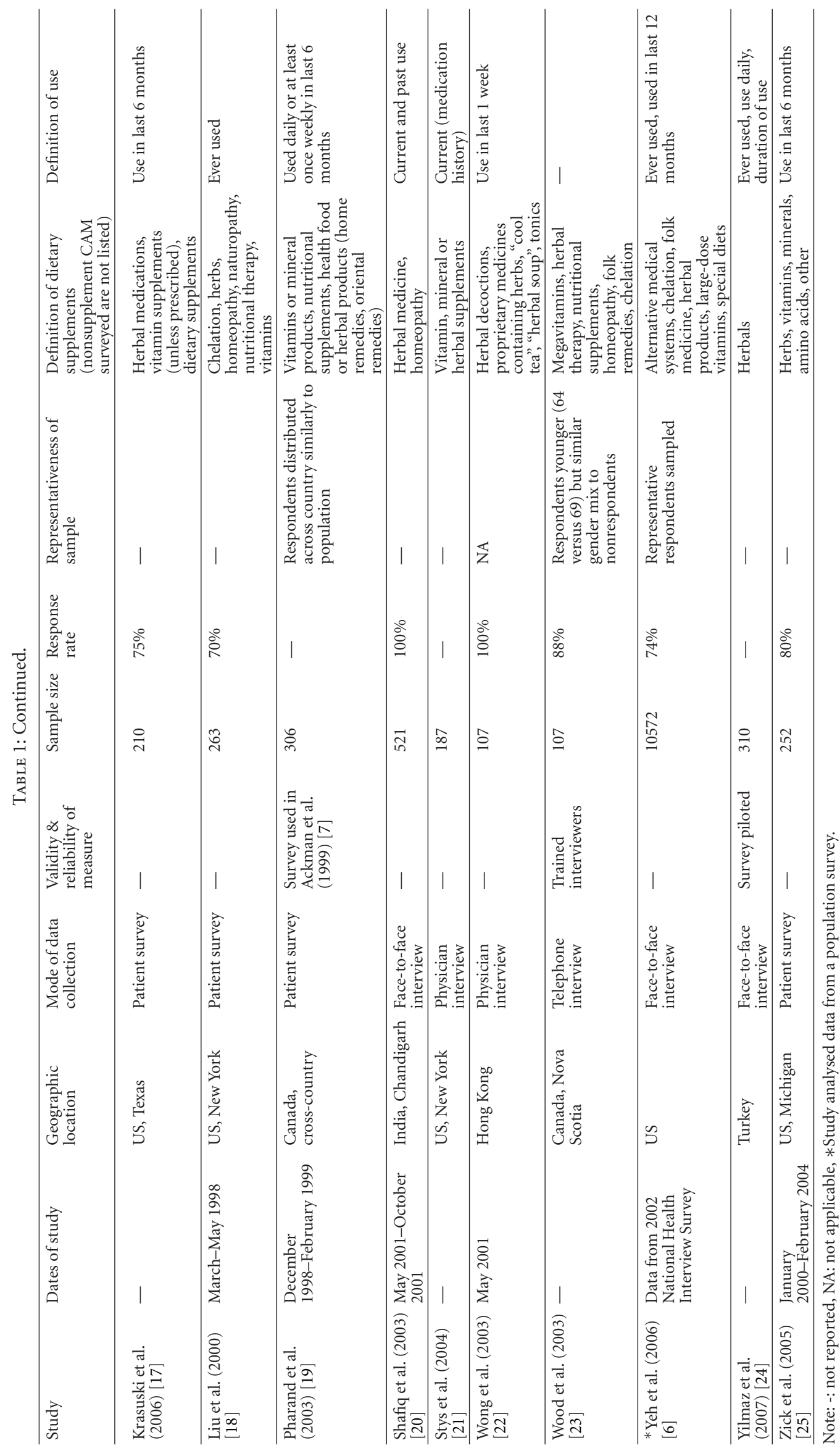




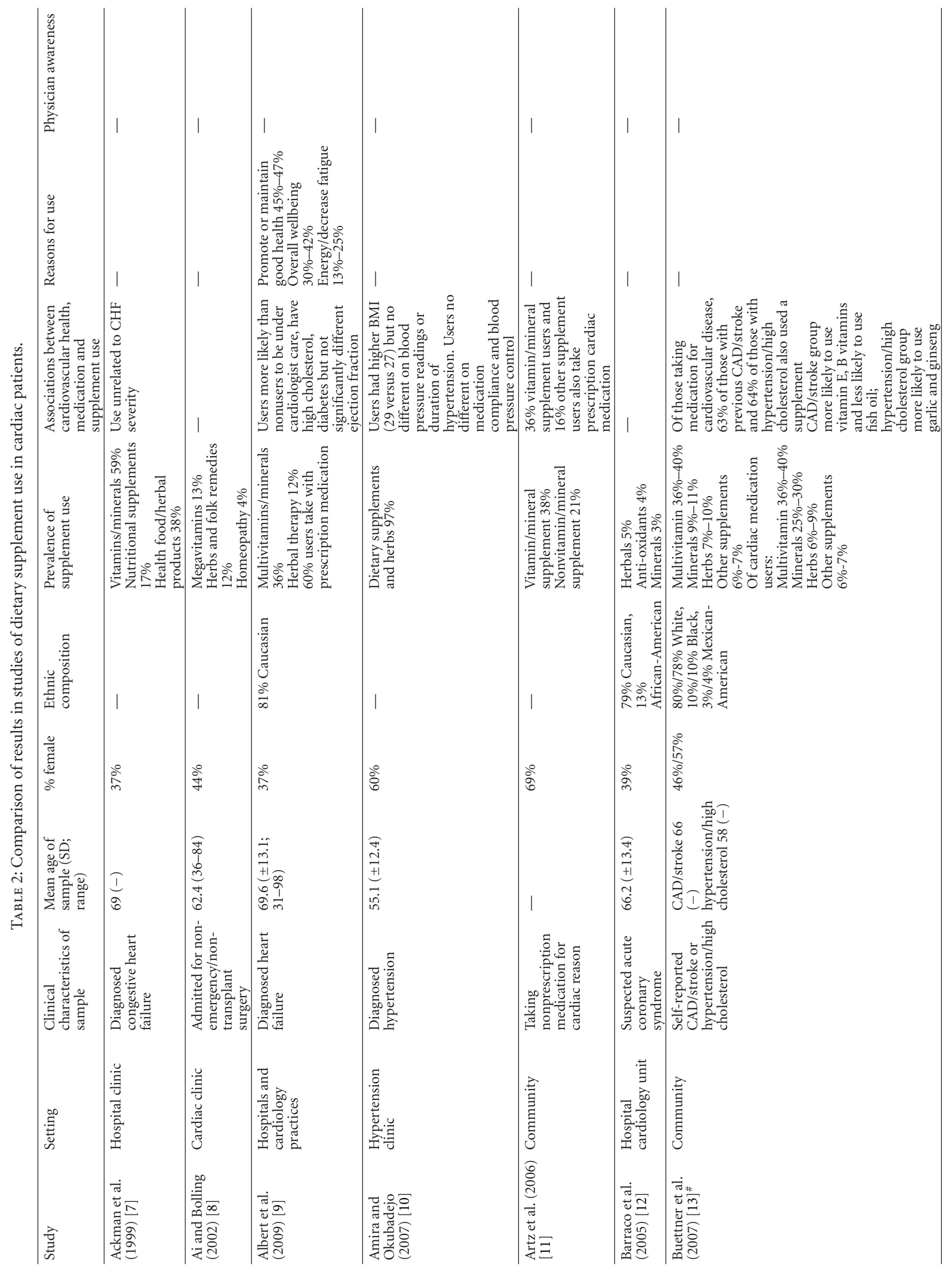




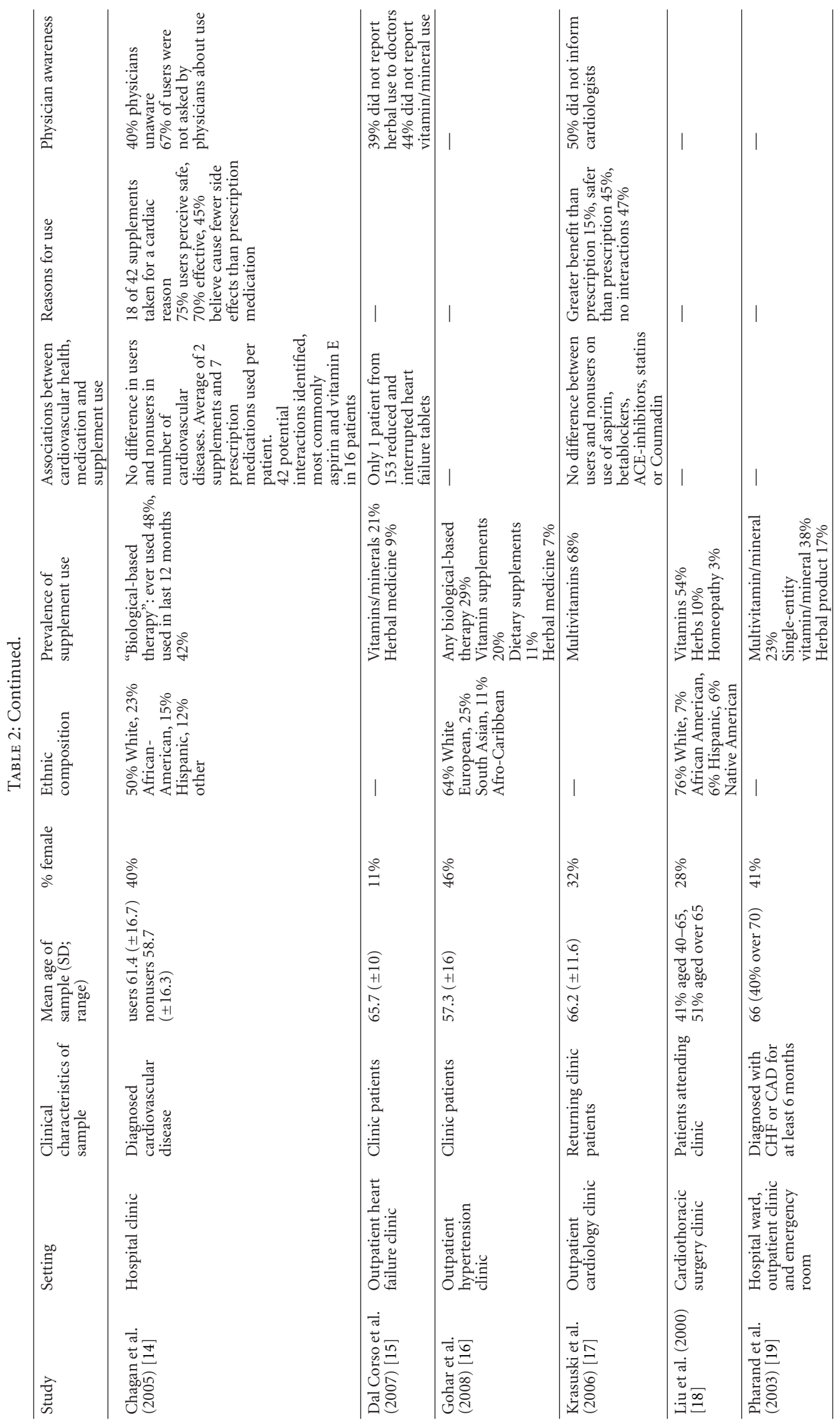




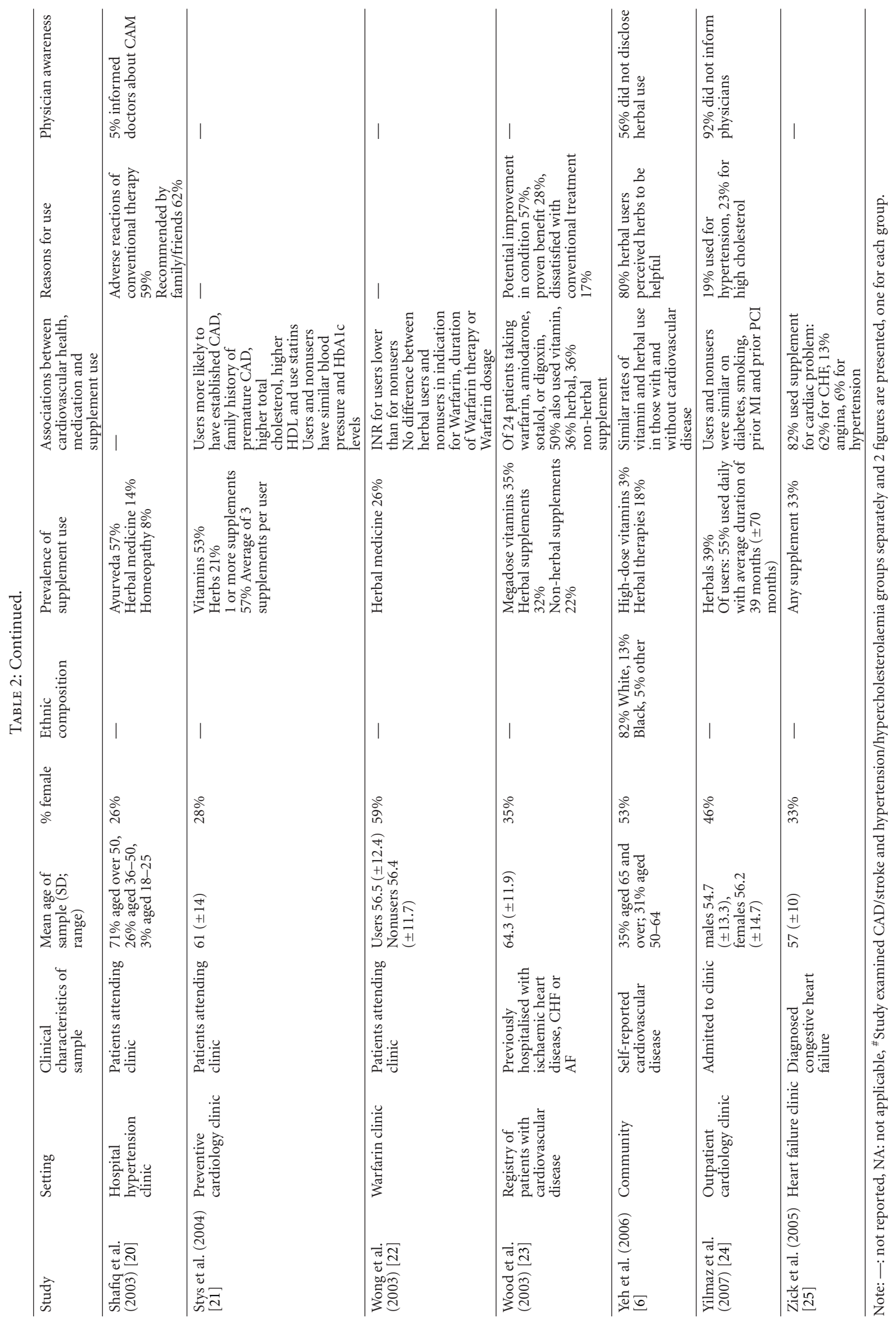


TABLE 3: Common supplements used by cardiac patients, pooled results from included studies.

\begin{tabular}{|c|c|c|c|c|}
\hline Supplement & Number of studies & Pooled $n$ & Pooled sample & Pooled prevalence $(\%)$ \\
\hline aloe vera & 2 & 15 & 423 & 3.6 \\
\hline Bilberry & 2 & 4 & 439 & 1.0 \\
\hline Calcium & 6 & 1,017 & 4,629 & 22.0 \\
\hline cayenne pepper/capsicum & 2 & 26 & 486 & 5.3 \\
\hline chromium/chromium picolinate & 3 & 11 & 679 & 1.6 \\
\hline coenzyme Q10 & 7 & 58 & 2,335 & 2.5 \\
\hline Dandelion & 2 & 4 & 359 & 1.0 \\
\hline Echinacea & 5 & 705 & 11,503 & 6.1 \\
\hline fish oil/omega-3 & 7 & 279 & 14,014 & 2.0 \\
\hline flax seed/flax seed oil & 5 & 48 & 1,235 & 3.9 \\
\hline folic acid & 7 & 160 & 5,108 & 3.1 \\
\hline Garlic & 12 & 902 & 16,417 & 5.5 \\
\hline Ginger & 6 & 302 & 11,909 & 2.5 \\
\hline ginkgo biloba & 6 & 572 & 15,131 & 3.8 \\
\hline Ginseng & 8 & 502 & 14,471 & 3.5 \\
\hline glucosamine/chondroitin & 8 & 499 & 11,855 & 4.2 \\
\hline green tea & 4 & 61 & 931 & 6.6 \\
\hline Hawthorn & 4 & 13 & 913 & 1.4 \\
\hline Iron & 4 & 26 & 673 & 3.9 \\
\hline Kelp & 2 & 7 & 486 & 1.4 \\
\hline Lecithin & 3 & 8 & 492 & 1.6 \\
\hline multivitamins and minerals & 9 & 1,883 & 5,525 & 34.1 \\
\hline Magnesium & 6 & 50 & 1,352 & 3.7 \\
\hline mint/lemon balm & 2 & 44 & 417 & 10.7 \\
\hline Nettle & 3 & 68 & 864 & 7.9 \\
\hline Parsley & 4 & 214 & 10,824 & 2.0 \\
\hline Peppermint & 2 & 214 & 10,824 & 2.0 \\
\hline Potassium & 3 & 87 & 4,034 & 2.2 \\
\hline Sage & 2 & 29 & 417 & 7.0 \\
\hline saw palmetto & 4 & 32 & 1,023 & 3.1 \\
\hline Selenium & 2 & 8 & 385 & 2.0 \\
\hline St John's wort & 3 & 220 & 11,053 & 2.0 \\
\hline Valerian & 3 & 24 & 882 & 2.7 \\
\hline vitamin A/beta-carotene & 6 & 107 & 4,671 & 2.3 \\
\hline vitamin B/B12/B complex & 8 & 470 & 5,261 & 8.9 \\
\hline vitamin $\mathrm{C}$ & 9 & 768 & 5,408 & 14.2 \\
\hline vitamin D & 4 & 42 & 1,047 & 4.0 \\
\hline vitamin $\mathrm{E}$ & 8 & 1,056 & 5,318 & 19.9 \\
\hline Zinc & 5 & 37 & 1,123 & 3.3 \\
\hline
\end{tabular}

Note: Only supplements with reported $\geq 1 \%$ use in 2 or more studies were included. Some figures were calculated from reported percentages.

did find higher supplement use in those with significant CAD (defined as prior myocardial infarction or previous percutaneous coronary intervention), and the studies of Ai and Bolling $[8,26]$ showed that use of herbs and folk remedies were significantly associated with arrhythmia and left main disease. Six studies specifically investigated patients with congestive heart failure (CHF) [7, 14, 15, 19, 23, 25]. In this population, supplement use was reported to be $40 \%$ [23] and $82 \%$ [25] although use appeared unrelated to the severity of CHF [7] or left ventricular ejection fraction $[9,21]$.
Four studies reported data on measurable outcomes. In Chinese patients taking warfarin, INR was significantly lower in herbal users than nonusers [22]. In two separate studies $[10,21]$ of clinic outpatients, blood pressure control did not differ between supplement users and nonusers although herbal use was associated with lower blood pressure in elderly patients with heart failure [9]. In one clinic population, lowdensity lipoprotein (LDL) levels also did not differ between supplement users and nonusers but high-density lipoprotein (HDL) was higher among the supplement users [21]. 
3.5. Patient Perceptions of Dietary Supplements. Seven studies investigated patient perceptions of dietary supplements $[6$, $9,14,17,20,23,24]$. The most common reasons for supplement use were: to promote or maintain health (up to $47 \%$ ), for overall wellbeing (up to $42 \%$ ), and for energy (up to 25\%) [9]. Dissatisfaction with conventional treatment was cited by $17 \%$ of patients in one study as a reason for supplement use [23] while in another, 59\% cited fear of adverse drug reactions as the reason for using CAM [20]. Three times more supplement users than nonusers thought supplements were safe (75\% versus 26\%) [14], with $45 \%$ of users believing supplements were safer than prescription medications [15], 30\% indicating that supplements were less likely to interact with other medicines [17], and 45\%$47 \%$ thinking that supplements would result in fewer side effects compared to prescription medications [14, 17]. Two studies found that $47 \%$ [17] and $81 \%$ [24] of users, respectively, were unaware herbal medicines could impact negatively on prescription medications. Dietary supplements were viewed as effective by $70 \%-80 \%$ of supplement users $[6,14]$.

Only one study uncovered negative views of CAM [20], finding that $56 \%$ of patients were dissatisfied with CAM. Two thirds of these patients thought CAM was too expensive and the rest believed that CAM caused adverse effects. The same study reported exacerbated hypertension made up 74\% of the adverse effects attributed by patients to CAM use [20] and all patients who reported adverse effects subsequently discontinued CAM use.

3.6. Physician Awareness of Patients' Supplement Use. Six studies asked whether patients informed doctors about supplement use and found physicians were not notified $44 \%$ (IQR $40 \%-53 \%$ ) of the time $[6,14,15,17,20,24]$. One study reported that $67 \%$ of supplement users did not disclose supplement use because their doctors did not ask [14], but other studies did not investigate the reasons behind nondisclosure.

\section{Discussion}

This is the first systematic review of dietary supplement use in cardiac patients and the findings have strong implications for clinical practice. The results indicate that supplement use is common in cardiac patients $(26 \%-42 \%)$ and that the concomitant use of dietary supplements and prescription medicine also appears to be frequent (16\%-64\%). Sicker patients may be more likely to seek out alternative treatments $[8,13,21]$, and drug interaction could play a causal role in the lower INR observed among supplement users on warfarin [22] or in the lower blood pressure among cardiac failure patients [9].

The potential for negative interaction in supplement users is high. Substances such as fish oil, hawthorn, garlic, ginseng, ginkgo, glucosamine, and parsley have antiplatelet properties $[27,28]$ and may interact with prescription antiplatelets or anticoagulants. Supplements such as capsicum and ginseng have been shown to affect blood pressure [29]. Supplemental potassium was taken by 1 in 5 respondents in one study [7]; this may result in adverse outcomes when used in combination with commonly prescribed cardiovascular medications such as angiotensin converting enzyme inhibitors, aldosterone receptor antagonists, or angiotensin receptor blockers. Other researchers have thoroughly reviewed the effects of herbal medicines and dietary supplements for cardiovascular health [30] as well as their potential for drug interaction [31] and we refer clinicians to these resources for more details.

It is worth noting that patients are likely to be using more than one supplement at a time [21], for periods of months to years $[13,24]$. This raises concerns of possible ongoing adverse interaction between supplements and prescription medications, as well as the potential for negative effects on compliance with conventional therapy. Many patients believe supplements are safe, effective, and produce few side effects. Many are also unaware that supplements can negatively impact prescription medications $[14,15,24]$. Moreover, a large proportion of physicians (39\%-95\%) are unaware of supplement use by their patients. Although patients may be reluctant to disclose supplement use, the main reason for nondisclosure appears to be physicians not asking about supplement use [14]. This suggests physicians can produce significant change in this aspect by being more proactive and asking about supplement use.

There are inconsistent results with regard to the association between supplement use and compliance. There is also limited evidence on whether supplement use affects clinical outcomes such as blood pressure and cholesterol control. The available evidence suggests that supplement use is associated with differences in health status of cardiac patients, although it is unknown whether supplement use causes these differences or whether differences in health cause patients to self-manage through supplementation.

The aggregated results presented here should be treated with caution. Our results show wide variability in the prevalence of dietary supplement use, which is consistent with other reviews of the CAM literature [32, 33]. Some of these variations may be due to the country and year in which the study was conducted. For the North American studies, the range of figures is smaller than for all studies but still large. For vitamin/mineral use it is 38\% (IQR 2649), for herbals 10\% (IQR 7-18), and for other supplements $7 \%$ (IQR $5 \%-13 \%$ ). In terms of variations over time, rates of supplement use seem to be decreasing over time, the overall rates being negatively correlated to the publication year in the North American studies $(r=-0.30)$. However, there are also severe methodological limitations in the quality and comparability of the reviewed studies. For instance, even though many of the included studies involved clinical samples, few provided objective data on the health status of the patients. Clinical diagnoses of cardiovascular disease were reported in only seven studies [7, 9, 10, $14,19,23,25]$, and only six compared supplement users and nonusers on clinical outcomes [7, 9, 10, 17, 21, 22]. Study comparison was also difficult due to heterogeneity in the definitions of dietary supplements and supplement use. For instance, some studies did not include common 
vitamins and supplements in their surveys, which would radically reduce the rates of supplement use. Some studies provided a checklist of supplements with no room for respondents to list other substances they may have used. Across studies, patients were asked whether they had used dietary supplements in the past year $[6,8,12,14,16]$, in the past month [13], in the past 3 and 6 months [7, 17, $19,25]$, as well as whether ever used in lifetime $[15,18]$. Two studies defined regular use as "at least once weekly" $[7,19]$ and only one asked for the duration of use [24]. An obvious result of using dissimilar definitions are the disparate prevalence rates even in the largest population studies, all conducted in North America $[6,11,13]$, which found supplement use to be $36 \%, 61 \%$, and $22 \%$, respectively.

Future studies should focus on developing three main areas. The first is to standardise and make explicit the definitions of dietary supplements and supplement use, which will facilitate cross-study comparison [33]. The second is to examine the impact of supplement use on prescription medications. Our paper found inconsistent associations between supplement use and compliance with prescription medications. Given that long-term compliance is essential to cardiac care, investigation into whether supplement use disrupts compliance is warranted. This is particularly important in population groups susceptible to noncompliance. In addition, the reluctance of patients to disclose use of dietary supplements may require patient and physician education. Lastly, studies are needed to examine both health and financial costs of supplement use. Only one of the reviewed studies examined the relative cost of CAM, finding that patients of an Indian clinic were spending the same amount on CAM and antihypertensive treatment, although the authors note that the cost of mainstream care can be reimbursed in most cases whilst CAM treatment cannot [20]. Prospective studies can assess whether concomitant use of prescription medications and supplements in cardiac patients affects outcomes such as morbidity, mortality, and quality of life, while economic evaluation can determine whether supplement use is cost effective, especially given the wide availability, increasing accessibility, and now common use of dietary supplements.

In summary, the use of dietary supplements is common in patients with cardiovascular conditions. There are no conclusive findings with respect to the health factors associated with this use although many commonly used supplements have the potential to interfere with the intended action of prescription medications. A substantial number of treating physicians are unaware of patients' supplement use, and consequently the effects of supplementation on conventional treatment may be overlooked. Disclosure of supplement use can be facilitated if medical practitioners are more proactive in questioning patients.

\section{Acknowledgments}

The authors would like to thank Probal Roy and Suzanne Grant for their invaluable comments on drafts of this paper.

\section{References}

[1] P. Kurtzweil, "An FDA guide to dietary supplements," FDA consumer, vol. 32, no. 5, pp. 28-35, 1998.

[2] P. M. Barnes, B. Bloom, and R. L. Nahin, "Complementary and alternative medicine use among adults and children: United States, 2007," National health statistics reports, no. 12, pp. 123, 2009.

[3] F. L. Bishop and G. T. Lewith, "Who uses CAM? a narrative review of demographic characteristics and health factors associated with CAM use," Evidence-Based Complementary and Alternative Medicine, vol. 7, no. 1, pp. 11-28, 2010.

[4] R. A. Bell, C. K. Suerken, J. G. Grzywacz, W. Lang, S. A. Quandt, and T. A. Arcury, "CAM use among older adults age 65 or older with hypertension in the United States: general use and disease treatment," Journal of Alternative and Complementary Medicine, vol. 12, no. 9, pp. 903-909, 2006.

[5] S. H. Shah, R. Engelhardt, and B. Ovbiagele, "Patterns of complementary and alternative medicine use among United States stroke survivors," Journal of the Neurological Sciences, vol. 271, no. 1-2, pp. 180-185, 2008.

[6] G. Y. Yeh, R. B. Davis, and R. S. Phillips, "Use of complementary therapies in patients with cardiovascular disease," American Journal of Cardiology, vol. 98, no. 5, pp. 673-680, 2006.

[7] M. L. Ackman, J. B. Campbell, K. A. Buzak, R. T. Tsuyuki, T. J. Montague, and T. Koon K, "Use of nonprescription medications by patients with congestive heart failure," Annals of Pharmacotherapy, vol. 33, no. 6, pp. 674-679, 1999.

[8] A. L. Ai and S. F. Bolling, "The use of complementary and alternative therapies among middle-aged and older cardiac patients," American Journal of Medical Quality, vol. 17, no. 1, pp. 21-27, 2002.

[9] N. M. Albert, L. Rathman, D. Ross et al., "Predictors of overthe-counter drug and herbal therapies use in elderly patients with heart failure," Journal of Cardiac Failure, vol. 15, no. 7, pp. 600-606, 2009.

[10] O. C. Amira and N. U. Okubadejo, "Frequency of complementary and alternative medicine utilization in hypertensive patients attending an urban tertiary care centre in Nigeria," BMC Complementary and Alternative Medicine, vol. 7, article no. 30, 2007.

[11] M. B. Artz, L. J. Harnack, S. J. Duval, C. Armstrong, D. K. Arnett, and R. V. Luepker, "Use of nonprescription medications for perceived cardiovascular health," American Journal of Preventive Medicine, vol. 30, no. 1, pp. 78-81, 2006.

[12] D. Barraco, G. Valencia, A. L. Riba, S. Nareddy, C. B. S. N. Draus, and S. M. Schwartz, "Complementary and alternative medicine (CAM) use patterns and disclosure to physicians in acute coronary syndromes patients," Complementary Therapies in Medicine, vol. 13, no. 1, pp. 34-40, 2005.

[13] C. Buettner, R. S. Phillips, R. B. Davis, P. Gardiner, and M. A. Mittleman, "Use of dietary supplements among United States adults with coronary artery disease and atherosclerotic risks," American Journal of Cardiology, vol. 99, no. 5, pp. 661-666, 2007.

[14] L. Chagan, D. Bernstein, J. W. M. Cheng et al., "Use of biological based therapy in patients with cardiovascular diseases in a university-hospital in New York City," BMC Complementary and Alternative Medicine, vol. 5, article no. 4, 2005.

[15] E. Dal Corso, A. L. Bondiani, L. Zanolla, and C. Vassanelli, "Nurse educational activity on non-prescription therapies in patients with chronic heart failure," European Journal of Cardiovascular Nursing, vol. 6, no. 4, pp. 314-320, 2007. 
[16] F. Gohar, S. M. Greenfield, D. Gareth Beevers, G. Y. H. Lip, and K. Jolly, "Self-care and adherence to medication: a survey in the hypertension outpatient clinic," BMC Complementary and Alternative Medicine, vol. 8, no. 1, article no. 4, 2008.

[17] R. A. Krasuski, K. Michaelis, and R. E. Eckart, "The cardiovascular patient's perceptions of complementary and alternative medicine," Clinical Cardiology, vol. 29, no. 4, pp. 161-164, 2006.

[18] E. H. Liu, L. M. Turner, S. X. Lin et al., "Use of alternative medicine by patients undergoing cardiac surgery," Journal of Thoracic and Cardiovascular Surgery, vol. 120, no. 2, pp. 335$341,2000$.

[19] C. Pharand, M. L. Ackman, C. A. Jackevicius, F. L. ParadisoHardy, and G. J. Pearson, "Use of OTC and herbal products in patients with cardiovascular disease," Annals of Pharmacotherapy, vol. 37, no. 6, pp. 899-904, 2003.

[20] N. Shafiq, M. Gupta, S. Kumari, and P. Pandhi, "Prevalence and pattern of use of complementary and alternative medicine (CAM) in hypertensive patients of a tertiary care center in India," International Journal of Clinical Pharmacology and Therapeutics, vol. 41, no. 7, pp. 294-298, 2003.

[21] T. Stys, A. Stys, P. Kelly, and W. Lawson, "Trends in use of herbal and nutritional supplements in cardiovascular patients," Clinical Cardiology, vol. 27, no. 2, pp. 87-90, 2004.

[22] R. S. M. Wong, G. Cheng, and T. Y. K. Chan, "Use of herbal medicines by patients receiving warfarin," Drug Safety, vol. 26, no. 8, pp. 585-588, 2003.

[23] M. J. Wood, R. L. Stewart, H. Merry, D. E. Johnstone, and J. L. Cox, "Use of complementary and alternative medical therapies in patients with cardiovascular disease," American Heart Journal, vol. 145, no. 5, pp. 806-812, 2003.

[24] M. B. Yilmaz, O. C. Yontar, O. O. Turgut et al., "Herbals in cardiovascular practice: are physicians neglecting anything?" International Journal of Cardiology, vol. 122, no. 1, pp. 48-51, 2007.

[25] S. M. Zick, A. Blume, and K. D. Aaronson, "The prevalence and pattern of complementary and alternative supplement use in individuals with chronic heart failure," Journal of Cardiac Failure, vol. 11, no. 8, pp. 586-589, 2005.

[26] A. L. Ai and S. F. Bolling, "Complementary and alternative care for the hearts of middle-aged and older patients," EvidenceBased Integrative Medicine, vol. 1, pp. 261-268, 2004.

[27] A. Fugh-Berman, "Herbs and dietary supplements in the prevention and treatment of cardiovascular disease," Preventive Cardiology, vol. 3, no. 1, pp. 24-32, 2000.

[28] P. Gardiner, R. Phillips, and A. F. Shaughnessy, "Herbal and dietary supplement-drug interactions in patients with chronic illnesses," American Family Physician, vol. 77, no. 1, pp. 73-80, 2008.

[29] C. Buettner, G. Y. Yeh, R. S. Phillips, M. A. Mittleman, and T. J. Kaptchuk, "Systematic review of the of ginseng on cardiovascular risk factors," Annals of Pharmacotherapy, vol. 40, no. 1, pp. 83-95, 2006.

[30] W. H. Frishman, P. Beravol, and C. Carosella, "Alternative and complementary medicine for preventing and treating cardiovascular disease," Disease-a-Month, vol. 55, no. 3, pp. 121-192, 2009.

[31] C. K. Vora and G. A. Mansoor, "Herbs and alternative therapies: relevance to hypertension and cardiovascular diseases," Current Hypertension Reports, vol. 7, no. 4, pp. 275-280, 2005.

[32] E. Ernst and B. R. Cassileth, "The prevalence of complementary/alternative medicine in cancer: a systematic review," Cancer, vol. 83, no. 4, pp. 777-782, 1998.
[33] P. Harris and R. Rees, "The prevalence of complementary and alternative medicine use among the general population: a systematic review of the literature," Complementary Therapies in Medicine, vol. 8, no. 2, pp. 88-96, 2000. 


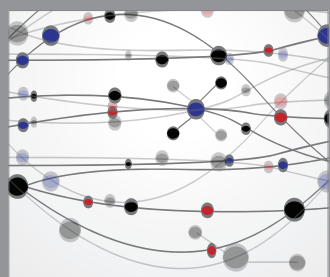

The Scientific World Journal
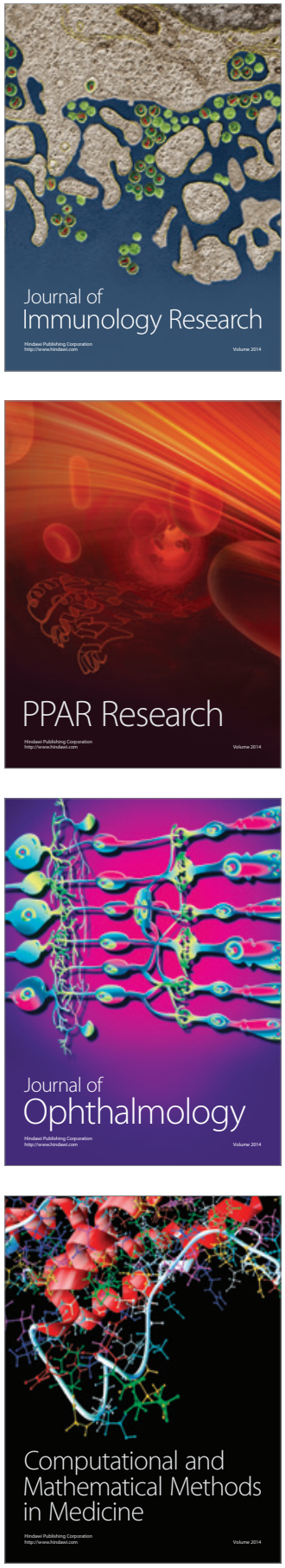

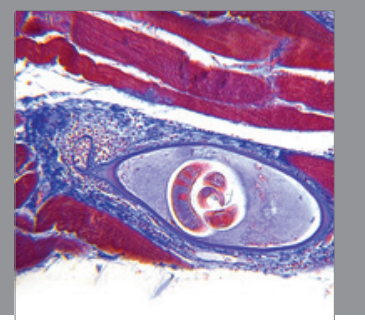

Gastroenterology

Research and Practice
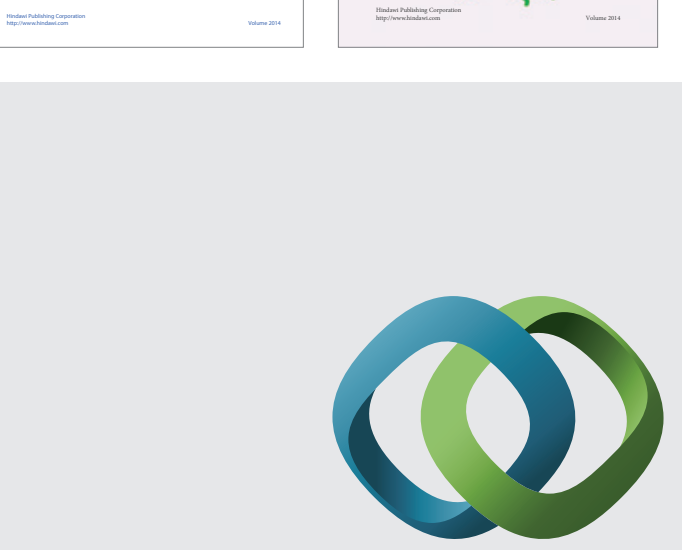

\section{Hindawi}

Submit your manuscripts at

http://www.hindawi.com
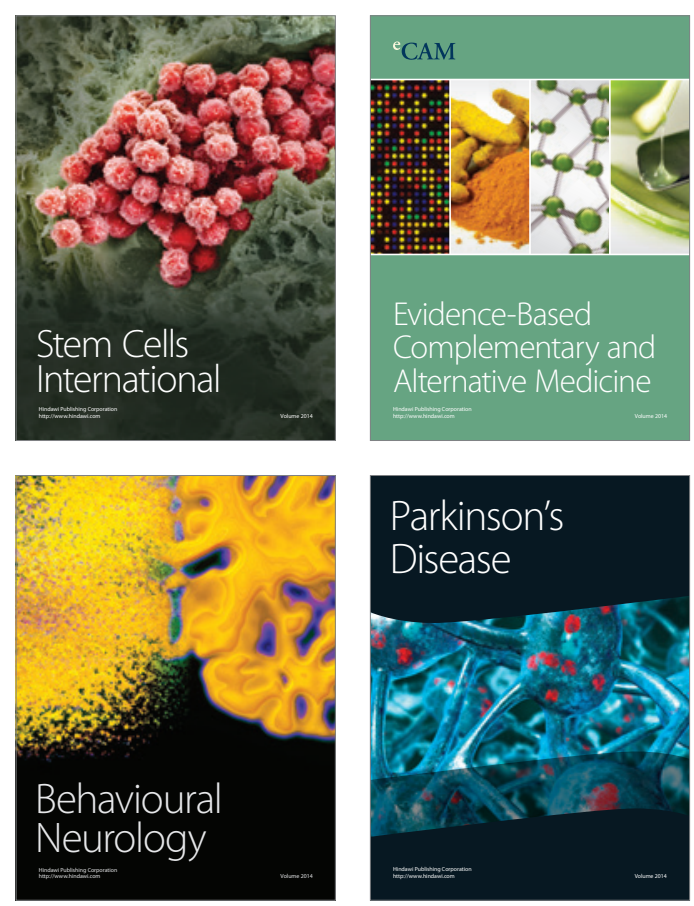

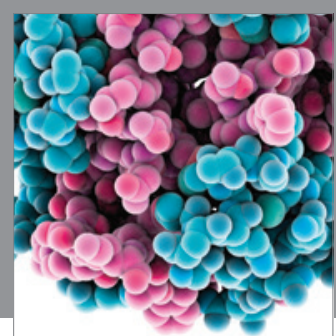

Journal of
Diabetes Research

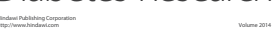

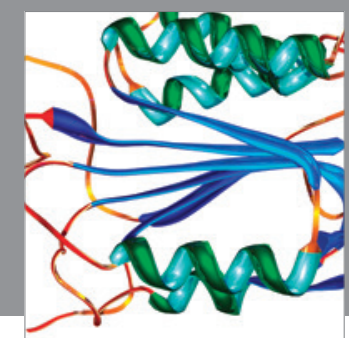

Disease Markers
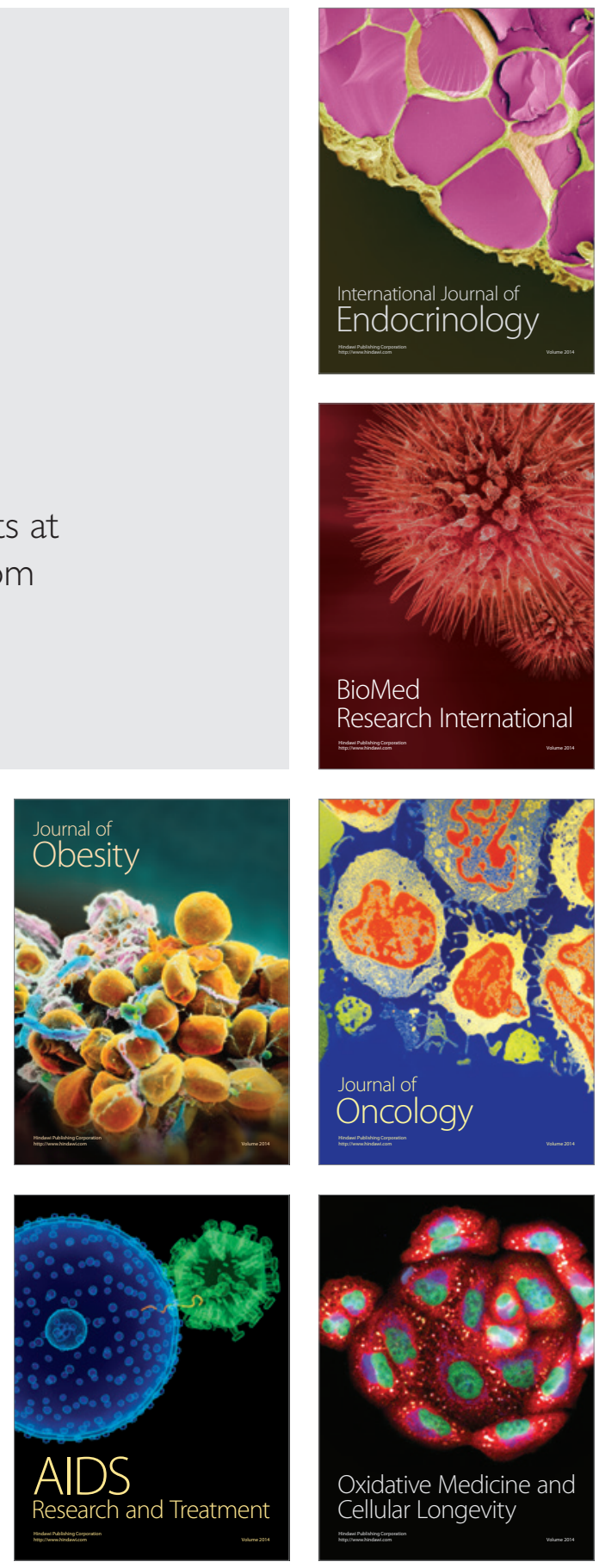